correct diagnosis. In the case of every patient who would formerly have been called neurasthenic, hysterical or psychasthenic, the thyroid function should be most minutely investigated, in order to ascertain whether this is not the cause of the existing neuro-endocrine disturbance.

M. Hamblin Smith.

\title{
4. Neurology.
}

An Improved Method of Encephalography. (Bull. Neur. Inst. N.Y., vol. ii, p. 75 , March, 1932.) Davidoff, L. M., and Dyke, C. G.

The routine is as follows: The evening before the day on which the procedure is to take place the patient is given sodium amytal, gr. iij, in order to ensure a restful night. This dose is repeated at $8 \mathrm{a} . \mathrm{m}$. At $8.45 \mathrm{a} . \mathrm{m}$. an injection of morphia is given (adults gr. $\frac{1}{6}$ to $\frac{1}{4}$, children gr. $1_{1}^{1}$ to $\frac{1}{2}$ ) and no breakfast. At 9 a.m. the operation is performed in the X-ray department. The patient sits on a bench with forehead resting against a vertical Potter-Bucky diaphragm, and lumbar puncture is performed under novocaine anæsthesia. On occasions when general anæsthesia is advisable, the same position of the patient is used during injection of air. To allow for the expansion of the air, an initial ro c.c. of cerebro-spinal fluid is removed and replaced by 5 c.c. of air. Thereafter 5 c.c. of air are introduced for every 5 c.c. of fluid removed. The fluid is removed by attaching a ro c.c. syringe to the lumbar puncture needle by means of a length of rubber tubing. Atmospheric air for injection is sucked into the syringe through many thicknesses of sterile gauze to exclude bacteria. Both removal of fluid and injection of air is done slowly, and the fluid is not sucked out, but allowed to fill 5 c.c. of the syringe "against the weight of the plunger with the syringe in a horizontal position".

Details as to amount of air to be injected and indication for greater or less volume are given together with details of radiographic technique. Stereoscopic films from four sides are taken in every case. The symptoms likely to arise during and after the procedure are given, and include headache, nausea, vomiting, cyanosis, pallor, perspiration, chilliness, drowsiness, restlessness and poor pulse. This procedure had been in use for two years in over 300 cases at the time of writing.

J. L. FAULL.

The Demonstration of Normal Cerebral Structures by Means of Encephalography. (Bull. Neur. Inst. N.Y., vol. ii, p.33 I, July, 1932.) Dyke, C.G., and Davidoff, L. M.

(1) The Choroid Plexuses.

This is the first of a series of studies upon the appearance of normal cerebral structures as demonstrated by the method of encephalography previously described by the authors. They claim to have demonstrated that certain defects in the bodies of the lateral ventricle and the roof of the fourth ventricle as outlined by air are caused by the choroid plexuses. The presence of such defects depends on the size of the choroid plexuses, and does not appear to be associated with any particular condition.

(Bull. Neur. Inst. N.Y., vol. iii, p. 138, June, 1933.)

(2) The Corpora Quadrigemina.

A group of one hundred encephalograms was reviewed to determine the frequency with which the corpora quadrigemina could be demonstrated. They were visible in $71 \%$ of the cases. This identification of these structures is possible by their size and shape and their fixed relation to other known structures of the brain that are easily recognized in the encephalogram. 
(Bull. Neur. Inst. N.Y., vol. iii, p. 147, June, 1933.)

(3) The Cerebral Convolutions and Sulci.

In a review of 700 encephalograms special attention was given to the identification of the various cerebral fissures, sulci and convolutions These structures on the medial aspect of the hemisphere are extremely easy to recognize. Beginning with the landmark of the corpus callosum, all the major sulci and convolutions and some of the minor ones are readily demonstrable. On the 1ateral surface of the cerebral hemisphere the convolutional pattern is much more complex, and a considerable amount of distortion occurs in the flat encephalogram as a result of the convexity of this surface. It is possible by stereoscopic films and the use of cranio-cerebral topography (after Taylor and Haughton) to recognize, first, the Sylvian and Rolandic fissures; following the identification of these cerebral markings and using them as landmarks, many of the other sulci and convolutions can be distinguished. By the use of illustration in stereoscopic pairs, the effort is made to convey the identity and three dimensional relations of the convolutions and sulci to the reader.

J. L. FAUll.

The Dangers and the Mortality of Ventriculography. (Bull. Neur. Inst. N.Y., vol. iii, p. 2 10, June, 1933.) Riggs, H.W.

The author undertook a study of 148 cases where the diagnostic procedure of ventriculography had been undertaken in the Neurological Institute, New York, in order to investigate the mortality-rate attending the operation. There were 12 deaths $(8 \cdot 1 \%)$. Most of the fatal cases occurred in patients with advanced symptoms and signs of intracranial tumour. Nearly all the patients who did badly after ventriculography, and in whom the situation of the lesion was verified by autopsy ( 7 cases) or at operation, had growths so situated as to be able profoundly to influence the third ventricle and the brain-stem with any change in pressure relations, such as were likely to result from the evacuation of fluid and the introduction of air. Dangerous symptoms followed by recovery occurred in $3 \mathbf{I}$ patients $(21 \%)$; in 23 of these the growths were deeply situated. Dangerous symptoms are due chiefly to a profound disturbance of the balance of pressure in the cranial cavity, and the frequency of their occurrence is not proportionate to the degree of dilatation of the ventricles or the amount of increase of intracranial pressure. Puncture of the ventricle and release of air will sometimes effect relief of symptoms; caffeine and hypertonic saline intravenously rarely proved beneficial. The author considers ventriculography to be a valuable and even indispensable diagnostic procedure, but it should be used only in those patients in whom localization of the tumour is impossible or uncertain by clinical means. J. L. FaUlL.

The Disturbances in Vision and in Visual Fields after Ventriculography. (Bull. Neur. Inst. N.Y., vol. iii, p. 190, June, 1933.) Masson, C. B.

The occurrence of temporary blindness after ventriculography in a patient in the Neurological Institute led the author to examine Ioo consecutive cases of ventriculography in order to learn how frequently disturbances in vision and in the visual fields occurred after the intraventricular introduction of air and what causative factor was involved. Six cases of temporary blindness occurred. Case-notes of each instance are given, together with details of technique and course of case. All the cases recovered sight within twenty-one to seventy-two hours. Possible causes are discussed, such as site and nature of cerebral lesion, track of needle, etc., but the author confesses himself unable to offer a satisfactory explanation for the occurrence of the symptoms.

J. L. FAULL.

Special Disability in Writing. (Bull. Neur. Inst. N.Y., vol. iii, p. I, June, I933.) Orton, S. T., and Gillingham, $A$.

Writing, in common with other fractions of the language faculty, is intimately related to the problem of unilateral cerebral dominance, since it is lost in lesions 